\title{
Significant oral cancer risk associated with low socioeconomic status
}

\author{
Abstracted from \\ Conway DI, Petticrew M, Marlborough H, Berthiller J, Hashibe M, Macpherson LM. \\ Socioeconomic inequalities and oral cancer risk: a systematic review and meta-analysis of case-control studies. \\ Int J Cancer 2008; 122: 2811-2819 \\ Address for correspondence: Dr. D. I. Conway, Dental Public Health Unit, University of Glasgow Dental School, \\ 378 Sauchiehall Street, Glasgow G2 3JZ, Scotland, UK. E-mail: d.conway@dental.gla.ac.uk
}

\section{Question: Is low socioeconomic status associated with increased oral cancer risk?}

Data Sources Searches were made for studies in Medline, Medline In-Process and other Non-indexed Citations Embase, CINAHL, PsychINFO, CAB Abstracts 1973-date, EBM Reviews, ACP Journal Club, Cochrane Register of Controlled Trials, Cochrane Database of Systematic Reviews, Database of Abstracts of Reviews of Effects, Health Management Information Consortium database and Pubmed. Un-published data were also received from the International Head and Neck Cancer Epidemiology Consortium.

Study selection Studies were identified independently by two reviewers and were included if their subject was oral and/ or oropharyngeal cancer; they used case-control methodology; gave data regarding socioeconomic status (SES; eg, educational attainment, occupational social classification or income) for both cases and controls; and the odds ratio (OR) for any SES measure was presented or could be calculated. Corresponding authors were contacted where there was an indication that data on oral and/ or oropharyngeal cancers could potentially be obtained from the wider cancer definition or grouping presented in the article, or if SES data were collected but had not been presented in the article. Methodological assessment of selected studies was undertaken. Data extraction and synthesis Countries where the study was undertaken were classified according to level of development and income as defined by the World Bank. Where available the adjusted OR (or crude OR) with corresponding $95 \%$ confidence intervals (Cl) were extracted, or were calculated for low compared with high SES categories. Meta-analyses were performed on the following subgroups: SES measure, age, sex, global region, development level, time-period and lifestyle factor adjustments. Sensitivity analyses were conducted based on study methodological issues. Publication bias was assessed using a funnel plot.

Results Forty-one studies met the inclusion criteria and yielded 15344 cases and 33852 controls. Compared with individuals who were in high SES strata, the pooled OR for the risk of developing oral cancer were 1.85 (95\% Cl, 1.60-2.15; $\mathrm{N}=37$ studies) for individuals with low educational attainment versus $1.84(95 \% \mathrm{Cl}, 1.47-2.31 ; \mathrm{N}=14)$ for those with low occupational social class versus and 2.41 (95\% Cl, 1.59$3.65 ; \mathrm{N}=5$ ) for people with low incomes. Subgroup analyses showed that low SES was significantly associated with increased oral cancer risk in high- and lower-income countries, across the world, and remained when adjusting for potential behavioural confounders.

Conclusions Oral cancer risk associated with low SES is significant and related to lifestyle risk factors. These results provide evidence to steer

\section{Commentary}

Major risk factors for oral cancer include tobacco, alcohol misuse and chewing areca nut (betel quid). Increased consumption of fruits and vegetables reduces oral cancer risk whereas human papillomavirus infection may contribute to an increased risk. Until recently the independent role of socioeconomic inequalities had not been investigated in detail. This systematic review examines whether oral cancer risk is associated with low SES.

The systematic review is well designed and sets out a clear focused question. Forty-one case-control studies were included in this metaanalysis: 24 of these were from high-income countries and 17 were from low-income countries. Three different measures were employed to define socioeconomic status: low income, low occupational social class and low educational attainment. By using all three measures in the inclusion criteria the authors accounted for the variations in SES measurements encountered in different studies reported in the literature.

It is interesting that, individually, each of the SES measures showed slightly different magnitudes of oral cancer risks, with OR ranging from 1.84-2.42. More studies $(\mathrm{N}=7)$ had used educational attainment as a measure, but the most significant risk of oral cancer was associated with low income.

Notably, four out of the 37 studies that provided data on the association of education with oral cancer risk concluded that high educational levels were associated with an increased risk for oral cancer. The authors provided some study characteristics (Table 2) and it can be seen these four studies were typical of other studies although some had rather small case numbers. There is evidence that among groups of young people affected by oral cancer (compared with older people) there is over-representation of subjects in high occupational social classes. ${ }^{1}$

Regular consumption of fruits and vegetables tends to be more rare in people with low incomes. The authors' subgroup analysis did not include information on any adjustment for nutrition which could have a significant effect on these estimates. ${ }^{2}$

The authors note the limited uniformity of data presentation in many of the reported studies. There were huge inter-study variations in classifying subjects. For example, in lower income countries, "no education" was compared with "ever education", whereas in highincome countries "high school education" was compared with "university education". This highlights an important issue when variables describing SES are allocated in study protocols. SES data gathering should be carefully planned to allow useful conclusions to be drawn from demographic studies. 
In addition to major risk factors for oral cancer (tobacco, alcohol and betel quid use), SES is also an important determinant of risk. This meta-analysis provides robust evidence that SES is an independent risk factor following adjustments for potential behavioural confounders. We could therefore speculate that the macro environment associated with low SES, such as the effect of poor education on health, lack of access to healthcare, hygiene, poor nutrition, unfavourable working environment and poor living conditions may contribute to causation of oral cancer by complex interactions in society, in synergy with the other known risk behaviours often encountered in any low SES groups.

This meta-analysis does make a significant contribution to the oral cancer literature in that it identifies low SES as an independent variable associated with an increased cancer risk. In addition to its utility for healthcare agencies interested in the control of oral can- cer, this meta-analysis provides useful data for the global partnerships and agencies that aim to reduce the poverty gap, and will help drive the case for better targeted and prioritised research to address the health needs of poor populations.

Saman Warnakulasuriya

Department of Oral Medicine, World Health Organization Collaborating Centre for Oral Cancer, King's College London Dental Institute, London, UK

1. Llewellyn CD, Linklater K, Bell J, Johnson NW, Warnakulasuriya S. An analysis of risk factors for oral cancer in young people: a case-control study. Oral Oncol 2004; 40: 304-313

2. Warnakulasuriya S. Food, nutrition and oral cancer. In Food Constituents and Oral Health. Edited by M Wilson. Cambridge: Woodhead; 2009: pp273-295.

Evidence-Based Dentistry (2009) 10, 4-5. doi:10.1038/sj.ebd.6400623 